Pacific Journal of Mathematics

LIPCHITZ SPACES ON THE SURFACE OF THE UNIT SPHERE 


\title{
LIPSCHITZ SPACES ON THE SURFACE OF THE UNIT SPHERE IN EUCLIDEAN $n$-SPACE
}

\author{
HARVEy C. GREenWALD
}

\begin{abstract}
This paper is concerned with defining Lipschitz spaces on $\Sigma_{n-1}$, the surface of the unit sphere in $R^{n}$. The importance of this example is that $\Sigma_{n-1}$ is not a group but a symmetric space. One begins with functions in $L_{p}\left(\Sigma_{n-1}\right), 1 \leqq p \leqq \infty . \quad \Sigma_{n-1}$ is a symmetric space and is related in a natural way to the rotation group $\mathrm{SO}(n)$. One can then use the group $\mathrm{SO}(n)$ to define first and second differences for functions in $L_{p}\left(\Sigma_{n-1}\right)$. Such a function is the boundary value of its Poisson integral. This enables one to work with functions which are harmonic. Differences can then be replaced by derivatives.
\end{abstract}

For a brief historical survey of Lipschitz spaces, the reader is referred to the introduction in Taibleson [18] and to the papers of Nikolskii [9] and Peetre [10]. For this paper, the approach of two people stands out as being of significant importance.

The first is Zygmund [20; Chapter VII]. Zygmund draws upon the results of Hardy and Littlewood [6]. For brevity we consider only the case $0<\alpha<1$. Let $f \in L_{p}[0,2 \pi]$ and be extended periodically, $1 \leqq p<\infty$, and let

$$
\omega_{p}(\delta)=\sup _{0<h<o}\left\{\frac{1}{2 \pi} \int_{0}^{2 \pi}|f(x+h)-f(x)|^{p} d x\right\}^{1 / p}
$$

Then $f \in \Lambda_{\alpha}^{p}$ if and only if $\omega_{p}(\delta)=O\left(\delta^{\alpha}\right)$. For $p=\infty$, let $\omega_{\infty}(\delta)=\sup$ $\left|f\left(x_{2}\right)-f\left(x_{1}\right)\right|$ where the sup is over all $x_{1}, x_{2}$ such that $\left|x_{1}-x_{2}\right| \leqq \delta$. Then $f \in \Lambda_{\alpha}^{\infty}$ if and only if $\omega_{\infty}(\delta)=O\left(\delta^{\alpha}\right)$.

An important result is that $u(r, x)$ is the Poisson integral of a function $f \in \Lambda_{\alpha}^{\infty}$ if and only if $(\partial / \partial x) u(r, x)=O\left(\delta^{\alpha-1}\right)$ where $\delta=1-r$, uniformly in $x$ as $r \rightarrow 1^{-}$.

The second person is Taibleson [18]. For brevity we consider only the case $0<\alpha<1$. Let $f \in L_{p}\left(\boldsymbol{R}^{n}\right), 1 \leqq p \leqq \infty$, and let $\|f(x+h)-f(x)\|_{p, d x}$ be the $L_{p}$ norm of $[f(x+h)-f(x)]$ considered as a function of $x$. Then $f \in \Lambda(\alpha ; p, q), 1 \leqq q<\infty$, if and only if

$$
\left\{\int_{\mathbb{R}^{n}}\left[|h|^{-\alpha}\|f(x+h)-f(x)\|_{p, d x}\right]^{q} d h /|h|^{n}\right\}^{1 / q}<\infty .
$$

An important result is that $f(x, y), 0<y<\infty$, is the Poisson integral of a function $f \in \Lambda(\alpha ; p, q)$ if and only if 


$$
\begin{aligned}
& \left\{\int_{0}^{\infty}\left[y^{1-\alpha}\left\|\frac{d}{d y} f(x, y)\right\|_{p, d x}\right]^{q} \frac{d y}{y}\right\}^{1 / q}+\|f\|_{p}<\infty \text { for } \\
& \quad 1 \leqq q<\infty, \sup _{y} y^{1-\infty}\left\|\frac{d}{d y} f(x, y)\right\|_{p, d x}+\|f\|_{p}<\infty \text { for } q=\infty .
\end{aligned}
$$

Some of the results in this paper are contained in a paper of Heideman [7]. Heideman deals with a generalization of these notions. $\mathrm{He}$ considers Banach spaces of distributions.

Another paper of interest is one by Stein [16] where he has worked out the important Littlewood-Paley theory for a compact Lie group.

Lastly, Ragozin [11] also has defined Lipschitz spaces on $\Sigma_{n-1}$. His notion is somewhat different and he is concerned mainly with polynomial approximation.

For the most part, the approach used in this paper is similar to Taibleson's approach as developed in [18], [19], and [20].

\section{Preliminaries.}

Definition. The Poisson kernel is the function $P(r x, y)=$ $C_{n}\left(1-r^{2}\right) /|r x-y|^{n}$ where $x, y \in \Sigma_{n-1}=\left\{x \in \boldsymbol{R}^{n}:|x|=1\right\}, 0 \leqq r<1$, and $C_{n}$ is a constant such that $\int_{\Sigma_{n-1}} P(r x, y) d y=1$ for each $x \in \Sigma_{n-1}$ where $d y$ is non normalized Lebesgue measure.

Note. It is often convenient to consider $P$ to be a function of $r$ and $\theta, 0 \leqq \theta \leqq 2 \pi$. Then

$$
P(r, \theta)=C_{n} \frac{1-r^{2}}{\left[1-2 r \cos \theta+r^{2}\right]^{n / 2}}=P(r, \cos \theta)
$$

where $\cos \theta=x \cdot y$. We shall also write

$$
P(r, x \cdot y)=C_{n} \frac{1-r^{2}}{\left[1-2 r x \cdot y+r^{2}\right]^{n / 2}} .
$$

Definition. Let $f \in L_{p}\left(\Sigma_{n-1}\right), 1 \leqq p \leqq \infty$. The Poisson integral of $f$ is defined as $f(r x)=\int_{\Sigma_{n-1}} f(y) P(r, x \cdot y) d y$. We shall assume that the reader is familiar with the properties of Poisson integrals. The reader is referred to Zygmund [21] for the one dimensional case and Stein and Weiss [17; Chapter 2] for the case of $\boldsymbol{R}^{n}$.

Notation. Let $Y_{l}^{(k)}, l=1, \cdots, n(k)$, be an orthonormal basis for the spherical harmonics of degree $k$. Let $Z_{y}^{(k)}$ be the zonal harmonic of degree $k$ with pole $y$. For a full discussion of spherical harmonics see Stein and Weiss [17; Chapter 4]. 
The following facts are well known:

$$
\begin{aligned}
& P(r, x \cdot y)=\sum_{k=0}^{\infty} r^{k} Z_{y}^{(k)}(x), \\
& f(r x)=\sum_{k=0}^{\infty} \sum_{l=1}^{n(k)} a_{k l} r^{k} Y_{l}^{(k)}(x),
\end{aligned}
$$

and the convergence is absolute and uniform for $r \leqq r_{0}<1$.

Definition. Let $F \in L_{1}\left(\Sigma_{n-1}\right)$ and $G \in L_{1}([-1,1], d \mu)$ where $d \mu(s)=\omega_{n-1}\left(1-s^{2}\right)^{(n-3) / 2} d s$ for $-1 \leqq s \leqq 1$ and $\omega_{n-1}$ is the surface area of $\Sigma_{n-1}$. The spherical convolution of $F$ and $G$ is the function

$$
F^{*} G(x)=\int_{\Sigma_{n-1}} F(y) G(x \cdot y) d y .
$$

(1.1) Let $F \in L_{p}\left(\Sigma_{n-1}\right)$ and $G \in L_{q}([-1,1], d \mu)$ where $0 \leqq 1 / p+1 / q-$ $1=1 / t \leqq 1$ and $1 \leqq p, q \leqq \infty$. Let $H(x)=\int_{\Sigma_{n-1}} F(y) G(x \cdot y) d y$. Then $H \in L_{t}\left(\Sigma_{n-1}\right)$ and $\|H\|_{t} \leqq\|F\|_{p}\|G\|_{q, d \mu}$.

REMARK. This is a standard result known as Young's inequality. See Calderón and Zygmund [4] or Askey and Wainger [1] for a more extensive discussion of spherical convolution.

(1.2) Let $f(r)$ be a nonnegative function defined on $0<r<1, \alpha \neq 0$, $p \geqq 1$. Let $F(s)$ be defined by $F(s)=\int_{0}^{s} f(r) d r$ if $\alpha>0$ and $F(s)=\int_{s}^{1} f(r) d r$ if $\alpha<0$. Then

$$
\begin{aligned}
& {\left[\int_{0}^{1}\left[(1-r)^{\alpha} F(r)\right]^{p} d r /(1-r)\right]^{1 / p} \leqq|\alpha|^{-1}} \\
& \quad \times\left[\int_{0}^{1}\left[(1-r)^{\alpha+1} f(r)\right]^{p} d r /(1-r)\right]^{1 / p} .
\end{aligned}
$$

REMARK. This is a standard result known as Hardy's inequality. See Hardy, Littlewood, and Połya [6] or Taibleson [18, Lemma 3, p. 418].

2. Radial derivatives. In this section Lipschitz spaces are defined. A justification for calling these spaces Lipschitz spaces will be given in $\S 4$.

There are two questions to be decided. The first is which differential operator to use. The simplest would be $\partial / \partial r$. Unfortunately, $f_{r}(r x)$ is not harmonic in general. However, $r f_{r}(r x)$ and $\partial / \partial r(r f)(r x)$ are harmonic. $r f_{r}(r x)$ has the disadvantage that the constant term in the expansion $f(r x)=\sum_{k, l} \alpha_{k l} r^{k} Y_{l}^{(k)}(x)$ is lost. Furthermore, the operator $(r f)_{r}$ is related to the Bessel potential operator. Thus, the operator $(r f)_{r}$ will be the one most often used. However, there will be circum- 
stances in which the other operators will be useful. For this reason the relations between these operators will be studied in this section.

The second question is: Which mixed norms are to be used? There are essentially two approaches. One uses Zygmund [21; Chapter VII] as a starting point and the other uses Taibleson [18] as a starting point.

The Taibleson norm

$$
\left[\int_{0}^{\infty}\left[y^{1-\alpha}\left\|\frac{\partial}{\partial y} f(x, y)\right\|_{p, d x}\right]^{q} d y / y\right]^{1 / q}
$$

can be transformed to

$$
\left[\int_{0}^{1}\left[(\ln 1 / r)^{1-\alpha}\left\|r f_{r}(r x)\right\|_{p, d x}\right]^{q} \frac{d r}{r \ln 1 / r}\right]^{1 / q}
$$

with the change of variables $r=e^{-y}$ where $1 \leqq q<\infty$, and

$$
f(x, y)=\sum_{k, l} a_{k l}\left(e^{-y}\right)^{l k} Y_{l}^{k k}(x) .
$$

This approach also correspond to that of Stein [16] for a compact Lie group. (Note that $\Sigma_{n-1}$ is the symmetric space $\mathrm{SO}(n) / K$ where $\mathrm{SO}(n)$ is the group of rotations on $\boldsymbol{R}^{n}$ and $K$ is the stability group of $e_{1}$. $\left\{e_{1}, \cdots, e_{n}\right\}$ is the canonical base for $\boldsymbol{R}^{n}$. $\mathrm{SO}(n)$ is, of course, a compact Lie group.)

On the other hand, if one chooses Zygmund [20; Chapter VII] as a starting point one should choose

$$
\left[\int_{0}^{1}\left[(1-r)^{1-\alpha}\left\|r f_{r}(r x)\right\|_{p, d x}\right] d r /(1-r)\right]^{1 / q}
$$

as a norm where $1 \leqq p \leqq \infty$ and $1 \leqq q<\infty$.

We now proceed to investigate the relationship between these various norms.

Definition. Let $x \in \boldsymbol{R}^{n}, 0<r<1$, and $1 \leqq p, q \leqq \infty$. We define

$$
\|F(r x)\|_{p q}=\left\|\left[\|F(r x)\|_{p, d x}\right]\right\|_{q, d r /(1-r)}
$$

and

$$
\|F(r x)\|_{p q}^{\#}=\left\|\left[\|F(r x)\|_{p, d x}\right]\right\|_{q, d r / r l n 1 / r} .
$$

(2.1) Let $f \in L_{p}\left(\Sigma_{n-1}\right), 1 \leqq p \leqq \infty$. Then if $\alpha>0$ and $1 \leqq q \leqq \infty$, the following are equivalent:

$$
\begin{gathered}
\left\|(1-r)^{\alpha} T^{k} f(r x)\right\|_{p q}+\|f(x)\|_{p} \text { where } T f=(r f)_{r}, \\
\left\|(1-r)^{\alpha} \frac{\partial^{k}}{\partial r^{k}} f(r x)\right\|_{p q}+\|f(x)\|_{p},
\end{gathered}
$$




$$
\left\|(1-r)^{\alpha} T_{1}^{k} f(r x)\right\|_{p q}+\|f(x)\|_{p} \text { where } T_{1} f=r f_{r} \text {, }
$$

$$
\begin{gathered}
\left\|(\ln 1 / r)^{\alpha} T_{1}^{k} f(r x)\right\|_{p q}^{*}+\|f(x)\|_{p}, \\
\left\|(r \ln 1 / r)^{\alpha} T^{k} f(r x)\right\|_{p q}^{*}+\|f(x)\|_{p} .
\end{gathered}
$$

REMARK 2.2. Let $f(r x)$ be harmonic on $B_{n}=\left\{x \in \boldsymbol{R}^{n}:|x|<1\right\}$. Assume $f(0)=0$. Then if $\alpha>0$ and $1 \leqq p, q \leqq \infty$, the corresponding $p q$ norms of (2.1) are equivalent.

(2.3) Let $f \in L_{p}\left(\Sigma_{n-1}\right), 1 \leqq p \leqq \infty$. Then if $\alpha>0$ and $1 \leqq q \leqq \infty$ and if $\bar{\alpha}$ is the smallest integer greater than $\alpha$ and $k$ is any integer greater than $\alpha$, the following are equivalent:

$$
\left\|(1-r)^{k-\alpha} T^{k} f(r x)\right\|_{p q}
$$

and

$$
\left\|(1-r)^{\bar{\alpha}-\alpha} T^{\bar{\alpha}} f(r x)\right\|_{p q} .
$$

REMARK. The proofs of the above use (1.1) and (1.2). See Taibleson [18] for the analogues in $\boldsymbol{R}^{n}$.

Definition. Let $\alpha>0$ and $1 \leqq p, q \leqq \infty$. Then $\Lambda\left(\alpha ; p, q ; \Sigma_{n-1}\right)=$ $\Lambda(\alpha ; p, q)$ is defined to be the set of functions $f \in L_{p}\left(\Sigma_{n-1}\right)$ for which the norm $\|f\|_{\alpha ; p, q}=\left\|(1-r)^{\bar{\alpha}-\alpha} T^{\bar{\alpha}} f(r x)\right\|_{p q}+\|f(x)\|_{p}$ is finite.

3. Tangential derivatives. In this section globially defined differential operators on $\Sigma_{n-1}$ are discussed. The reader is referred to Ragozin [11] for a more complete discussion of these operators. Let $D$ be an $n \times n$ skew-symmetric matrix and let $f \in L_{1}\left(\Sigma_{n-1}\right)$. Define $D f(x)=\left.(d / d t) f[(\exp t D)(x)]\right|_{t=0}$. Since $d /\left.d t(\exp t D)(x)\right|_{t=0}=D x$, the map from the matrix $D$ to the differential operator $D$ is linear. Define an inner product by $\left\langle D_{1}, D_{2}\right\rangle=-1 / 2$ trace $\left(D_{1} D_{2}\right)$. Let $D_{i j}$ be the map which takes $e_{i}$ to $e_{j}, e_{j}$ to $-e_{i}$, and $e_{k}$ to zero if $k \neq i, j$ and $i<j$. Then $\left\{D_{i j}\right\}$ is an orthonormal basis for the skew-symmetric matrices. Actually this is an inner product on linear transforms since the trace is invariant under change of basis.

(3.1) Let $f(r x)$ be harmonic on $B_{n}$. Then if $\alpha>0$ and $1 \leqq p, q \leqq \infty$, the following are equivalent:

$$
\begin{gathered}
\left\|(1-r)^{\alpha} f_{r}(r x)\right\|_{p q}, \\
\sum_{i<j}\left\|(1-r)^{\alpha} D_{i j} f(r x)\right\|_{p q}, \\
\left\|(1-r)^{\alpha} \nabla_{T} f(r x)\right\|_{p q}
\end{gathered}
$$

where $\nabla_{T}$ is the gradient in the tangential plane for $f(r x)$ considered 
as a function of $x$.

REMARK. The proof is similar to the proofs of (2.1), (2.2), and (2.3). Again, the reader is referred to Taibleson [17].

4. First and second differences. In this section, first and second differences for functions with domain $\Sigma_{n-1}$ are defined. The equivalence between the norms based on differences and the norms introduced in $\S 2$ is shown. It is at this point that the symmetric space property of $\Sigma_{n-1}$ is used. $\Sigma_{n-1}$ can be identified with $\mathrm{SO}(n) / \mathrm{SO}(n-1)$.

For this chapter only, we assume that the Lebesgue measure $d y$ is normalized so that $\int_{\Sigma_{n-1}} d y=1$. We also normalize the Haar measure $d u$ on $\mathrm{SO}(n)$ so that $\int_{\mathrm{SO}(n)} d u=1$.

If $f$ is defined on $\Sigma_{n-1}$, we can construct a function $\widetilde{f}(u)=f\left[u\left(x_{0}\right)\right]$ where $x_{0} \in \Sigma_{n-1}$ and is fixed. Then

$$
\int_{\Sigma_{n-1}} f(y) d y=\int_{\mathrm{SO}(n)} \tilde{f}(u) d u=\int_{\mathrm{SO}(n)} f\left[u\left(x_{0}\right)\right] d u .
$$

Equality holds for any point $x_{0} \in \Sigma_{n-1}$. For a more extensive discussion of these notions see Coifman and Weiss [5]. This enables one to work with the group $\mathrm{SO}(n)$ in attempting to define differences.

We are going to decompose $\mathrm{SO}(n)$ into a "polar decomposition". Let $\rho \in \mathrm{SO}(n)$ be such that $\rho\left(e_{1}\right)=e_{1}$. Then by an abuse of notation we can consider $\rho$ to be in $\mathrm{SO}(n-1)$. For $u \in \mathrm{SO}(n)$, it is easily seen that the spherical distance between $\left(u \rho \exp t D_{12}\right)\left(e_{1}\right)$ and $(u \rho)\left(e_{1}\right)$ is $t$. [The spherical distance between $x, y \in \Sigma_{n-1}$ is defined by $d(x, y)=$ $\left.\cos ^{-1}(x \cdot y) \cdot\right]$

Definition. Let $u \in \mathrm{SO}(n), \quad \rho \in \mathrm{SO}(n-1), \quad 1 \leqq p, q \leqq \infty$, and $0<t<\pi$. We define

$$
\|G(u, t)\|_{p q}=\left\|\left(\|G(u, t)\|_{p, d u}\right)\right\|_{q, d t / t}
$$

and

$$
\begin{aligned}
\|H(u, \rho, t)\|_{p q}^{*} & =\left\{\int_{0}^{\pi} \int_{\operatorname{SO}(n-1)}\left(\|G(u, \rho, t)\|_{p, d u}\right)^{q} d \rho d t / t\right\}^{1 / q} \\
& \quad \text { if } 1 \leqq q<\infty \\
= & \sup _{0<t<\pi} \sup _{\rho \in \operatorname{SO}(n-1)}\|G(u, \rho, t)\|_{p, d u} \text { if } q=\infty .
\end{aligned}
$$

We consider the following first difference:

$$
\begin{aligned}
& \left\|t^{-\alpha}\left\{f\left[\left(u \exp t D_{12}\right)\left(e_{1}\right)\right]-f\left[u\left(e_{1}\right)\right]\right\}\right\|_{p q} \\
= & \left\|t^{-\alpha}\left\{f\left[\left(u \rho \exp t D_{12}\right)\left(e_{1}\right)\right]-f\left[u \rho\left(e_{1}\right)\right]\right\}\right\|_{p q}^{*} .
\end{aligned}
$$


Similarly we can define a second difference. The points $\left(u \rho \exp t D_{12}\right)\left(e_{1}\right),(u \rho)\left(e_{1}\right)$, and $\left(u \rho \exp -t D_{12}\right)\left(e_{1}\right)$ all lie on a great circle and are equally spaced with $(u \rho)\left(e_{1}\right)$ between $\left(u \rho \exp t D_{12}\right)\left(e_{1}\right)$ and $\left(u \rho \exp -t D_{12}\right)\left(e_{1}\right)$.

We consider the following second difference:

$$
\begin{aligned}
& \left\|t^{-\alpha}\left\{f\left[\left(u \exp t D_{12}\right)\left(e_{1}\right)\right]-2 f\left[u\left(e_{1}\right)\right]+f\left[\left(u \exp -t D_{12}\right)\left(e_{1}\right)\right]\right\}\right\|_{p q} \\
& \quad=\left\|t^{-\alpha}\left\{f\left[\left(u \rho \exp t D_{12}\right)\left(e_{1}\right)\right]-2 f\left[(u \rho)\left(e_{1}\right)\right]+f\left[\left(u \rho \exp -t D_{12}\right)\left(e_{1}\right)\right]\right\}\right\|_{p q}^{*}
\end{aligned}
$$

where $1 \leqq p, q \leqq \infty$ and $\alpha>0$.

We now proceed to establish the equivalence stated earlier.

Proposition 4.1. Let $f \in L_{p}\left(\Sigma_{n-1}\right), 1 \leqq p \leqq \infty$, and let $f(r x)$ be its Poisson integral. If $0<\alpha<1$ and $1 \leqq q \leqq \infty$, the following are equivalent:

$$
\begin{gathered}
\left\|(1-r)^{1-\alpha} r f_{r}(r x)\right\|_{p q} \\
\| t^{-\alpha}\left\{f\left[\left(u \exp t D_{12}\right)\left(e_{1}\right)\right]-f\left[u\left(e_{1}\right)\right]\right\}_{p q}
\end{gathered}
$$

Proof. The proof is similar to the proof of the succeeding Proposition 4.3. First, write

$$
r f D_{r}(r x)=\int_{\mathrm{so}(n)}\left\{f\left[\left(u \rho \exp t D_{12}\right)\left(e_{1}\right)\right]-f\left[(u \rho)\left(e_{1}\right)\right]\right\} r P_{r}(r, \cos t) d v .
$$

One can now proceed as in Proposition 4.3 to get part of the result. For the other part write

$$
\begin{aligned}
& s f\left[s\left(u \exp t D_{12}\right)\left(e_{1}\right)\right]-s f\left[s u\left(e_{1}\right)\right] \\
& =\int_{r}^{s}(\sigma f)_{o}\left[\sigma\left(u \exp t D_{12}\right)\left(e_{1}\right)\right] d \sigma-\int_{r}^{s}(\sigma f)_{\sigma}\left[\sigma u\left(e_{1}\right)\right] d \sigma \\
& \quad+r f\left[r\left(u \exp t D_{12}\right)\left(e_{1}\right)\right]-r f\left[r u\left(e_{1}\right)\right]
\end{aligned}
$$

and note that

$$
f\left[r\left(u \exp t D_{12}\right)\left(e_{1}\right)\right]-f\left[r u\left(e_{1}\right)\right]=\int_{0}^{t} D f\left[r\left(u \exp s D_{12}\right)\left(e_{1}\right)\right] d s .
$$

The result now follows from (3.1).

LeMmA 4.2. Let $f \in \Lambda(\alpha ; p, q)$ where $\alpha>0$ and $1 \leqq p, q \leqq \propto$. Then $\left\|(r f)_{r}(r x)\right\|_{p, d x}=o(1 /(1-r))$ as $r \rightarrow 1^{-}$.

Proof. The proof is analogous to the proof of Lemma 5 of Taibleson [17; p. 426]. The proof uses $(2.3)$ and the fact that $\left\|(r f)_{r}(r x)\right\|_{p, d x}$ is increasing as a function of $r$. 
Proposition 4.3. Let $f \in L_{p}\left(\Sigma_{n-1}\right), 1 \leqq p \leqq \infty$, and $f(r x)$ its Poisson integral. If $0<\alpha<2$ and $1 \leqq q \leqq \infty$, the following are equivalent:

$$
\left\|(1-r)^{2-\alpha} r\left(r f_{r}\right)_{r}(r x)\right\|_{p q}
$$

(ii)

Proof. (a) (i) $\leqq A_{\alpha}$ (ii).

$$
f\left[u e_{1}\right]=\int_{\Sigma_{n-1}} f[u y] P\left(r y \cdot e_{1}\right) d y .
$$

Let $A$ be a linear transform on $\boldsymbol{R}^{n}$ defined by $A\left(e_{1}\right)=e_{1}$ and $A\left(e_{i}\right)=-e_{i}$ for $i=2, \cdots, n$. Then

$$
\begin{aligned}
f\left[u e_{1}\right] & =\int_{\Sigma_{n-1}} f[(u A) y] P\left(r A(y) \cdot e_{i}\right) d y \\
& =\int_{\Sigma_{n-1}} f[(u A)(y)] P\left(r y \cdot e_{1}\right) d y \\
& =\int_{\mathrm{So}(n)} f\left[(u A v)\left(e_{1}\right)\right] P\left(r v\left(e_{1}\right) \cdot e_{1}\right) d v .
\end{aligned}
$$

There exists a $\rho \in \mathrm{SO}(n)$ such that $\rho e_{1}=e_{1}$ and $\left(\rho \exp t D_{12}\right)\left(e_{1}\right)=v\left(e_{1}\right)$ where $\cos t=v\left(e_{1}\right) \cdot e_{1}$ and $\rho=\rho(v), t=t(v)$. Then $A(v)\left(e_{1}\right)=(A \rho \exp$ $\left.t D_{12}\right)\left(e_{1}\right)=\left(\rho \exp -t D_{12}\right)\left(e_{1}\right)$ since $\rho\left(e_{2}\right) \cdot e_{1}=0$. Therefore,

$$
f\left[u\left(e_{1}\right)\right]=\int_{\mathrm{so}(n)} f\left[\left(u \rho \exp -t D_{12}\right)\left(e_{1}\right)\right] P(r, \cos t) d v .
$$

Hence

$$
\begin{aligned}
2 r\left(r f_{r}\right)_{r}\left[r u\left(e_{1}\right)\right]= & \int_{\mathrm{so}(n)}\left\{f\left[\left(u \rho \exp t D_{12}\right)\left(e_{1}\right)\right]-2 f\left[u e_{1}\right]\right. \\
& \left.\left.+f\left[u \rho \exp -t D_{12}\right)\left(e_{1}\right)\right]\right\} r\left(r P_{r}\right)_{r}(r, \cos t) d v,
\end{aligned}
$$

since $d / d r \int_{\mathrm{SO}(n)} P(r, \cos t) d v=0$. Therefore,

$$
\begin{aligned}
& \left\|r\left(r f_{r}\right)_{r}(r x)\right\|_{p, d x}=\left\|r\left(r f_{r}\right)_{r}\left[r u\left(e_{1}\right)\right]\right\|_{p, d u} \\
& \leqq \\
& \quad \int_{\mathrm{SO}(n)} \| f\left[\left(u \rho \exp t D_{12}\right)\left(e_{1}\right)\right]-2 f\left[(u \rho)\left(e_{1}\right)\right] \\
& \quad+f\left[\left(u \rho \exp -t D_{12}\right)\left(e_{1}\right)\right] \|_{p, d u}\left|r\left(r P_{r}\right)_{r}(r, \cos t)\right| d v \\
& \quad=\int_{\mathrm{SO}(n)} g(t)\left|r\left(r P_{r}\right)_{r}(r, \cos t)\right| d v
\end{aligned}
$$

where

$$
g(t)=\left\|f\left[\left(u \exp t D_{12}\right)\left(e_{1}\right)\right]-2 f\left[u e_{1}\right]+f\left[\left(u \exp -t D_{12}\right)\left(e_{1}\right)\right]\right\|_{p, d u} .
$$

Case I. $q=\infty$. Let $A=\sup _{0<t<\pi} t^{-\alpha} g(t)$. Then 


$$
\begin{aligned}
& (1-r)^{2-\alpha}\left\|r\left(r f_{r}\right)_{r}(r x)\right\|_{p, d x} \\
& \quad \leqq(1-r)^{2-\alpha} A C_{n} \int_{0}^{\pi} t^{\alpha} \sin ^{n-2} t\left|r\left(r P_{r}\right)_{r}(r, \cos t)\right| d t
\end{aligned}
$$

by the previous inequality since the integrals over $\mathrm{SO}(n)$ and $\Sigma_{n-1}$ are related. The above is bounded by

$$
\begin{aligned}
& (1-r)^{2-\alpha} A C_{n} \int_{0}^{1-r} t^{\alpha} \sin ^{n-2} t\left|r\left(r P_{r}\right)_{r}(r, \cos t)\right| d t \\
& \quad+(1-r)^{2-\alpha} A C_{n} \int_{1-r}^{\pi} t^{\alpha} \sin ^{n-2} t\left|r\left(r P_{r}\right)_{r}(r, \cos t)\right| d t .
\end{aligned}
$$

The first term is bounded by

$$
\begin{aligned}
& (1-r)^{2-\alpha} A C_{n}^{\prime}(1-r)^{\alpha}(1-r)^{n-2}\left(1-r^{2}\right)(1-r)^{-n} \\
& \times(1-r)^{-2}(1-r)=2 C_{n}^{\prime} A
\end{aligned}
$$

since $\left|r\left(r P_{r}\right)_{r}(r, \cos t)\right| \leqq B(1-r)^{-2} P(r, \cos t)$. In as much as

$$
\left(\sin ^{n-2} t\right)\left[1-2 r \cos t+r^{2}\right]^{-n / 2} \leqq B t^{-2},
$$

the second term is bounded by

$$
(1-r)^{2-\alpha} A C_{n}^{\prime \prime} \int_{1-r}^{\pi} t^{\alpha}(1-r)^{-2}\left(1-r^{2}\right) B t^{-2} d t \leqq A B_{n} .
$$

Case II. $1 \leqq q \leqq \infty$. By an argument similar to the one used in Case I we have $(1-r)^{2-\alpha}\left\|r\left(r f_{r}\right)_{r}(r x)\right\|_{p, d x}$

$$
\begin{aligned}
\leqq & C_{n}(1-r)^{2-\alpha} \int_{0}^{(1-r) \pi}\left(\sin ^{n-2} t\right) g(t)\left|r\left(r P_{r}\right)_{r}(r, \cos t)\right| d t \\
& +C_{n}(1-r)^{2-\alpha} \int_{(1-r) \pi}^{\pi}\left(\sin ^{n-2} t\right) g(t)\left|r\left(r P_{r}\right)_{r}(r, \cos t)\right| d t .
\end{aligned}
$$

Hence, $\left\|(1-r)^{2-\alpha} r\left(r f_{r}\right)_{r}(r x)\right\|_{p q} \leqq I+I I$ where I and II are the $L_{q}([0,1], d r /(1-r))$ norms of the first and second terms respectively. Now $I \leqq$

$$
\begin{aligned}
C_{n}^{\prime}\left\{\int_{0}^{1}\left[(1-r)^{2-\alpha} \int_{0}^{1-r} \sin ^{n-2}(\pi t) g(\pi t)\left|r\left(r P_{r}\right)_{r}(r, \cos \pi t)\right| d t\right]^{q}\right. \\
\quad d r /(1-r)\}^{1 / q} \\
\quad \leqq C_{n}^{\prime \prime}\left\{\int_{0}^{1}\left[(1-r)^{2-\alpha} \int_{0}^{1-r} \sin ^{n-2}(\pi t) g(\pi t) P(r, \cos \pi t) d t\right]^{q} d r /(1-r)\right\}^{1 / q} .
\end{aligned}
$$

But $\left(\sin ^{n-2} \pi t\right)\left[1-2 r \cos \pi t+r^{2}\right]^{1-(n / 2)} \leqq 1$ and $(1-r)^{-1}[1-2 r \cos \pi t+$ $\left.r^{2}\right]^{-1} \leqq t^{-3}$ since $t \leqq(1-r)$. Hence I 


$$
\begin{aligned}
& \leqq 2 B_{n}^{\prime \prime}\left\{\int_{0}^{1}\left[(1-r)^{-\alpha} \int_{0}^{1-r} t^{-3} g(\pi t) d t\right]^{q} d r /(1-r)\right\}^{1 / a} \\
& \leqq B_{n, \alpha}\left\{\int_{0}^{1}\left[(1-r)^{-\alpha} g[(1-r) \pi]\right]^{q} d r /(1-r)\right\}^{1 / q}
\end{aligned}
$$

by (1.2). But this is equal to

$$
B_{n, \alpha}^{\prime}\left\{\int_{0}^{\pi}\left[t^{-\alpha} g(t)\right]^{\alpha} d t / t\right\}^{1 / q}
$$

By a similar argument

$$
\mathrm{II} \leqq K_{n}\left\{\int_{0}^{1}\left[(1-r)^{-\alpha} \int_{1-r}^{1} t^{-1} g(\pi t) d t\right]^{q} d r /(1-r)\right\}^{1 / q} .
$$

By (1.2) this is bounded by

$$
\begin{gathered}
K_{n, \alpha}\left\{\int_{0}^{1}\left[(1-r)^{-\alpha} g[\pi(1-r)]\right]^{q} d r /(1-r)\right\}^{1 / q} \\
=K_{n, \alpha}^{\prime}\left\{\int_{0}^{\pi}\left[t^{-\alpha} g(t)\right]^{q} d t / t\right\}^{1 / q} .
\end{gathered}
$$

(b) (ii) $\leqq A_{\alpha}(i)$. We may assume $f(0)=0$. Let $u \in \mathrm{SO}(n), 0<t<\pi$, and $r$ be such that $\pi(1-r)=t$.

$$
\begin{aligned}
s f(s x)= & \int_{r}^{s}(\rho f)_{\rho}(\rho x) d \rho+r f(r x) \\
= & \int_{r}^{s}(1-\rho)(\rho f)_{\rho \rho}(\rho x) d \rho-(1-s)(r f)_{r}(s x) \\
& +(1-r)(r f)_{r}(r x)+r f(r x) .
\end{aligned}
$$

Thus

$$
\begin{aligned}
t^{-\alpha} \| & s f\left[s\left(u \exp t D_{12}\right)\left(e_{1}\right)\right]-2 s f\left[s u\left(e_{1}\right)\right] \\
& +s f\left[s\left(u \exp -t D_{12}\right)\left(e_{1}\right)\right]\left\|_{p, d u} \leqq 4(1-s) t^{-\alpha}\right\|(r f)_{r}(s x) \|_{p, d x} \\
& +4 \pi^{\alpha}(1-r)^{-\alpha} \int_{r}^{1}(1-\rho)\left\|(\rho f)_{\rho \rho}(\rho x)\right\|_{p, d x} \\
& +2 \pi^{\alpha}(1-r)^{-\alpha+1}\left\|(r f)_{r}\left[r\left(u \exp t D_{12}\right)\left(e_{1}\right)\right]-(r f)_{r}\left[r u\left(e_{1}\right)\right]\right\|_{p, d u} \\
& +t^{-\alpha} \| r f\left[r\left(u \exp t D_{12}\right)\left(e_{1}\right)\right]-2 r f\left[r u\left(e_{1}\right)\right] \\
& +r f\left[r\left(u \exp -t D_{12}\right)\left(e_{1}\right)\right] \|_{p, d u} .
\end{aligned}
$$

(I) For the first, by Lemma 2.2 we have that

$$
\lim _{s \rightarrow 1^{-}}(1-s)\left\|(r f)_{r}(s x)\right\|_{p, d x}=0 .
$$

(II) The $L_{p}([0,1], d r /(1-r))$ norm of the second term is bounded 


$$
\begin{aligned}
& K\left\{\int_{0}^{1}\left[(1-r)^{-\alpha} \int_{r}^{1}(1-\rho)\left\|(\rho f)_{\rho \rho}(\rho x)\right\|_{p, d x} d \rho\right]^{q} d r /(1-r)\right\}^{1 / q} \\
& \quad \leqq K_{\alpha}\left\|(1-r)^{2-\alpha}(r f)_{r r}\right\|_{p q} \text { by (1.2) if } q<\infty .
\end{aligned}
$$

This is bounded by $K_{\alpha}^{\prime}\left\|(1-r)^{2-\alpha} T^{2} f(r x)\right\|_{p q}$ by Remark 2.2 since $f(0)=0$. The above is bounded by

$$
A_{\alpha}\left\|(1-r)^{2-\alpha} T^{2} f(r x)\right\|_{p q} \leqq B_{\alpha}\left\|(1-r)^{2-\alpha} r\left(r f_{r}\right)_{r}(r x)\right\|_{p q} .
$$

A similar argument applies if $q=\infty$.

(III) For the third term let $D=u D_{12} u^{-1}$.

$$
\begin{aligned}
(r f)_{r} & {\left[r\left(u \exp t D_{12}\right)\left(e_{1}\right)\right]-(r f)_{r}\left[u\left(e_{1}\right)\right] } \\
& =\int_{0}^{t} \frac{d}{d s}(r f)_{r}\left[r(\exp s D)\left(u\left(e_{1}\right)\right)\right] d s \\
& =\sum_{i<j} \int_{0}^{t} b_{i j} D_{i j}(r f)_{r}\left[r\left(u \exp s D_{12}\right)\left(e_{1}\right)\right] d s
\end{aligned}
$$

where $D=\sum_{i<j} b_{i j} D_{i j}$ and $\sum_{i<j} b_{i j}^{2}=1$.

Therefore,

$$
\begin{aligned}
& \left\|(r f)_{r}\left[r\left(u \exp t D_{12}\right)\left(e_{1}\right)\right]-(r f)_{r}\left[r u\left(e_{1}\right)\right]\right\|_{p, d u} \\
& \quad \leqq \sum_{i \leq j} t\left\|D_{i j}(r f)_{r}\left[r u\left(e_{1}\right)\right]\right\|_{p, d u} .
\end{aligned}
$$

Hence

$$
\begin{aligned}
& \left\|t^{-\alpha}(1-r)\left\{(r f)_{r}\left[r\left(u \exp t D_{12}\right)\left(e_{1}\right)\right]-(r f)_{r}\left[r u\left(e_{1}\right)\right]\right\}\right\|_{p q} \\
& \quad \leqq C_{\alpha} \sum_{i<j}\left\|(1-r)^{2-\alpha} D_{i j}(r f)_{p}(r x)\right\|_{p q} \\
& \quad \leqq A_{\alpha}\left\|(1-r)^{2-\alpha} T^{2} f(r x)\right\|_{p q} \leqq B_{\alpha}\left\|(1-r)^{2-\alpha} r\left(r f_{r}\right)_{r}(r x)\right\|_{p q}
\end{aligned}
$$

by (3.1) and Remark (2.2).

(IV) For the remaining term,

$$
\begin{gathered}
f\left[r\left(u \exp t D_{12}\right)\left(e_{1}\right)\right]-2 f\left[r u\left(e_{1}\right)\right]+f\left[r\left(u \exp -t D_{12}\right)\left(e_{1}\right)\right] \\
\quad=\int_{-t}^{0} \int_{0}^{t} \frac{d^{2}}{d \sigma d s} f\left[r(\exp (\sigma+s) D)\left(u\left(e_{1}\right)\right)\right] d s d \sigma
\end{gathered}
$$

with $D$ as in (III) above. This equals

$$
\sum_{l<k} \sum_{i<j} \int_{-t}^{0} \int_{0}^{t} b_{i j} b_{l k} D_{i j} D_{l k} f\left[r\left(u \exp (\sigma+s) D_{12}\right)\left(e_{1}\right)\right] d \sigma d s .
$$

Since $(1-r) \pi=t$,

$$
\begin{aligned}
& \left\|t^{-\alpha}\left\{f\left[r\left(u \exp t D_{12}\right)\left(e_{1}\right)\right]-2 f\left[r u\left(e_{1}\right)\right]+f\left[r\left(u \exp -t D_{12}\right)\left(e_{1}\right)\right]\right\}\right\|_{p q} \\
& \quad \leqq C \sum_{l<k} \sum_{i<j}\left\|t^{2-\alpha} D_{i j} D_{l k} f\left[r u\left(e_{1}\right)\right]_{p q} \leqq B \sum_{i<k} \sum_{i<j}\right\|(1-r)^{2-\alpha} D_{i j} D_{l k} f(r x) \|_{p q} .
\end{aligned}
$$

The above is bounded by 


$$
A_{\alpha, n} \sum_{l<k}\left\|(1-r)^{2-\alpha} D_{l k} r f_{r}(r x)\right\|_{p q} \leqq B_{\alpha, n}\left\|(1-r)^{2-\alpha} r\left(r f_{r}\right)_{r}(r x)\right\|_{p q} .
$$

This completes the proof of Proposition 4.3.

5. The Bessel potential operator. In this section the Bessel potential operator is defined and some of its more elementary properties are listed.

Proposition 5.1. Let $f(r x)$ be harmonic on $B_{n}$ and bounded for $0 \leqq r \leqq \rho$ where $0<\rho<1$. Suppose $\left\|(1-r)^{k-\alpha} T^{k} f(r x)\right\|_{p q} \leqq D$ for some $k>\alpha>0,1 \leqq p, q \leqq \infty$. Assume also that there exists an $r_{0}$ such that $0<r_{0}<1$ and $\|f(r x)\|_{p, d x} \leqq D$ for $0 \leqq r \leqq r_{0}$. Then $f(r x)$ is the Poisson integral of a function $f \in \Lambda(\alpha ; p, q)$ and

$$
\|f\|_{\alpha ; p, q} \leqq A_{\alpha, k, r_{0}} D \text {. }
$$

Proof. The proof is analogous to the proof of Lemma 5 of Taibleson [18; p. 426]. The proof uses Proposition 2.3.

Definition. Let $G^{\alpha}(r x)=\sum_{k=0}^{\infty} r^{k}(k+1)^{-\alpha} Z_{e_{1}}^{(k)}(x)$. For $\alpha>0$ this will turn out to be the Poisson integral of a function $G^{\alpha}(x) \in L_{1}\left(\Sigma_{n-1}\right)$. For $\alpha \leqq 0$ this will be the Poisson integral of a distribution. The case $\alpha \leqq 0$ will be discussed in a later paper.

REMARK. $G^{\alpha}$ will be used to define a multiplier $J^{\alpha}$ with the property that if $f \in L_{p}\left(\Sigma_{n-1}\right)$ and $f \sim \sum_{k, l} a_{k l} Y_{l}^{(k)}$, then

$$
J^{\alpha} f \sim \sum_{k, l} a_{k l}(k+1)^{-\alpha} Y_{l}^{(k)} .
$$

Multipliers similar to this have been investigated by Hirschman [8] for $L_{p}(0,2 \pi)$ and by Askey and Wainger [1] for $L_{p}\left(\Sigma_{n-1}\right)$.

Notation. Let $P_{k}^{\lambda}(t), \lambda>0,-1 \leqq t \leqq 1$, be the Gegenbauer polynomials defined by $\sum_{k=0}^{\infty} z^{k} P_{k}^{\lambda}(t)=\left(1-2 t z+z^{2}\right)^{-\lambda}$ for $|z|<1$. It is well known that $Z_{e_{1}}^{(k)}(x)=Z^{(k)}\left(x \cdot e_{1}\right)=C_{n}(k+\lambda) P_{k}^{\lambda}\left(x \cdot e_{1}\right)$ where $\lambda=(n-2) / 2$ and $C_{n}$ is a constant. The following facts will be useful in obtaining information about $G^{\alpha}(x)$.

(5.2) Let $\alpha>0$ and $t=x \cdot e_{1}$. Then

(i) $G^{\alpha}(r x)$ is the Poisson integral of a function $G^{\alpha}(x) \in L_{1}\left(\Sigma_{n-1}\right)$.

(ii) For $t \neq 1, G^{\alpha}(x)=\lim _{r \rightarrow 1^{-}} G^{\alpha}(r x)$ in the pointwise sense.

(iii) $G^{\alpha}(x)$ is continuous if $t \neq 1$.

(iv) If $t \neq 1$,

$$
\begin{array}{ll}
\left|G^{\alpha}(r x)\right| \leqq M_{\alpha, \lambda}(1-t)^{(\alpha-2 \lambda-1) / 2} & \text { if } \alpha<2 \lambda+1, \\
\left|G^{\alpha}(r x)\right| \leqq M_{\alpha, \lambda} \ln (1-t) & \text { if } \alpha=2 \lambda+1,
\end{array}
$$


where $M_{\alpha, \lambda}$ is a constant depending only on $\alpha$ and $\lambda$.

(v) If $\alpha>2 \lambda+1, G^{\alpha}(x)$ is continuous for all $x \in \Sigma_{n-1}$ and hence $G^{\alpha}(r x)$ is bounded for all $x \in \Sigma_{n-1}$.

Proof. See Askey and Wainger [1].

We shall also need some information about $G^{\alpha}(r x)$ when $-\infty<\alpha \leqq 0$.

(5.3) If $\lambda+1<\alpha<2 \lambda+1$ and $-1<t<1$, then

$$
\sum_{k=1}^{\infty}(k+\lambda) k^{-\alpha}\left|P_{k}^{\lambda}(t)\right| \leqq M_{\alpha, \lambda}\left(1-t^{2}\right)^{(\alpha-2 \lambda-1) / 2} .
$$

Proof. See Askey and Wainger [1; Lemma 5, p. 204].

(5.4) Let $F_{\varepsilon}(t)=\sum_{k=1}^{\infty}(k+\lambda)\left(e^{-\varepsilon}\right)^{k}(k+1)^{-\alpha} P_{k}^{\lambda}(t) \quad$ where $\varepsilon>0$ and $-\infty<\alpha<2 \lambda+1$. Then if $-1<t<1$,

$$
\left|F_{\varepsilon}(t)\right| \leqq M_{\alpha, \lambda}\left(1-t^{2}\right)^{(\alpha-2 \lambda-1) / 2} \text {. }
$$

Proof. Choose $\nu$ so large that $\alpha+2 \nu>(\lambda+\nu)+1$. By Askey and Wainger [1; p. 199, line 5]

$$
F_{\varepsilon}(t)=\sum_{j, l, m} \sum_{k=1}^{\infty} a_{j, k, l, m}\left(e^{-\varepsilon}\right)^{k} k^{-\nu-j+1} \varepsilon^{m} P_{k}^{\lambda+\nu}(t)\left[G_{l}(k)+G_{l+1}(k)\right]
$$

where $G_{l}(k)=\max _{k \leqq t \leqq k+b_{\nu}}\left|d^{l} / d t^{l}(1+t)^{-\alpha}\right|, a_{j, k, l, m}$ and $b_{\nu}$ are bounded, $l+j+m=\nu ; l, j, m$ are nonnegative integers, and $\nu-j \geqq 1$. If $\alpha$ is a nonpositive integer we assume that $-\alpha-l \geqq 0$. Since $\left(e^{-\varepsilon}\right)^{k} \varepsilon^{m}=$ $O\left(k^{-m}\right)$,

$$
\begin{aligned}
\left|F_{\varepsilon(t)}\right| & \leqq A_{\nu, \lambda} \sum_{j, l, m} \sum_{k=1}^{\infty} k^{-\nu-j+1} k^{-m}(k+1)^{-\alpha-1}\left|P_{k}^{\lambda+\nu}(t)\right| \\
& \leqq A_{\nu, \lambda} \sum_{k=1}^{\infty} k^{-2 \nu-\alpha+1}\left|P_{k}^{\lambda+\nu}(t)\right| \leqq M_{\nu, \lambda}\left(1-t^{2}\right)^{(\alpha-2 \lambda-1) / 2}
\end{aligned}
$$

by (5.3) for $-1<t<1$.

REMARK. If we set $r=e^{-\varepsilon}$, we have from the above that

$$
\left|G^{\alpha}(r x)\right| \leqq M_{\alpha, \lambda}\left(1-t^{2}\right)^{(\alpha-2 \lambda-1) / 2}
$$

if $-\infty<\alpha<2 \lambda+1$ for $t \neq \pm 1$ where $t=x \cdot e_{1}$.

$$
G^{\alpha}(x) \geqq 0 \text { if } \alpha>0 \text {. }
$$

Proof. In view of (5.2), it suffices to show that $G^{\alpha}(r x) \geqq 0$. $P\left(r x, e_{1}\right)=\sum_{k=0}^{\infty} r^{k} Z_{e_{1}}^{(k)}(x) \geqq 0$. Hence $\sum_{k=0}^{\infty} r^{k} e^{-t k} Z_{e_{1}}^{(k)}(x) \geqq 0$ and so

$$
\begin{aligned}
0 & \leqq \Gamma(\alpha)^{-1} \int_{0}^{\infty} t^{\alpha} e^{-t}\left(\sum_{k=0}^{\infty} r^{k} e^{-t k} Z_{e_{1}}^{(k)}(x)\right) d t / t \\
& =\Gamma(\alpha)^{-1} \sum_{k=0}^{\infty} r^{k} Z_{e_{1}}^{(k)}(x) \int_{0}^{\infty} t^{\alpha-1} e^{-t-t k} d t .
\end{aligned}
$$


This is justified since the series converges absolutely and uniformly for $0 \leqq r<1$. The above is equal to

$$
\begin{gathered}
\sum_{k=0}^{\infty} r^{k} Z_{e_{1}}^{(k)}(x) \Gamma(\alpha)^{-1} \int_{0}^{\infty} t^{\alpha-1} e^{-(k+1) t} d t \\
=\sum_{k=0}^{\infty} r^{k} Z_{e_{1}}^{(k)}(x) \Gamma(\alpha)^{-1} \int_{0}^{\infty} \frac{s^{\alpha-1}}{(k+1)^{\alpha-1}} e^{-s} \frac{d s}{k+1} \\
=\sum_{k=0}^{\infty} r^{k} Z_{e_{1}}^{(k)}(x)(k+1)^{-\alpha}=G^{\alpha}(r x) . \\
\int_{\Sigma_{n-1}} G^{\alpha}(x) d x=1 \text { if } \alpha>0 . \\
G^{\alpha_{1}+\alpha_{2}}(x)=G^{\alpha_{1} *} G^{\alpha_{2}}(x) \text { if } \alpha_{1}, \alpha_{2}>0 .
\end{gathered}
$$

Proof. (5.6) and (5.7) are clear since $G^{\alpha}(r x) \rightarrow G^{\alpha}(x)$ as $r \rightarrow 1^{-}$in $L_{1}\left(\Sigma_{n-1}\right)$.

$$
G^{\alpha}(x) \in \Lambda\left(\alpha-\frac{(n-1)}{p^{\prime}} ; p, \infty\right) \text { if } 0<\alpha-\frac{(n-1)}{p^{\prime}}<1 .
$$

Proof. Let $\lambda=(n-2) / 2$. We are going to apply Proposition 5.1 to $G^{\alpha}(r x)$,

(a) Suppose $\alpha<2 \lambda+2 . \quad G^{\alpha}(r x)=\sum_{k=0}^{\infty}(k+1)^{-\alpha} Z_{e_{1}}^{(k)}(x)$ and

$$
T G^{\alpha}(r x)=\sum_{k=0}^{\infty}(k+1)^{1-\alpha} Z_{e_{1}}^{(k)}(x)=G^{\alpha-1}(r x) .
$$

By (5.4) with $t=x \cdot e_{1}$, if $t \neq \pm 1,\left|T G^{\alpha}(r x)\right| \leqq M_{\alpha, n}\left(1-t^{2}\right)^{(\alpha-2 \lambda-2) / 2}$. Thus if $p<\infty,\left\|T G^{\alpha}(r x)\right\|_{p q}$

$$
\begin{aligned}
& \leqq M_{\alpha, n}^{\prime}\left[\int_{-1+(1-r)^{2}}^{1-(1-r)^{2}}\left|T G^{\alpha}(r, t)\right|^{p}\left(1-t^{2}\right)^{\lambda-1 / 2} d t\right]^{1 / p} \\
& +M_{\alpha, n}^{\prime}\left[\int_{1-(1-r)^{2}}^{1}\left|T G^{\alpha}(r, t)\right|^{p}\left(1-t^{2}\right)^{\lambda-1 / 2} d t\right]^{1 / p} \\
& +M_{\alpha, n}^{\prime}\left[\int_{-1}^{-1+(1-r)^{2}}\left|T G^{\alpha}(r, t)\right|^{p}\left(1-t^{2}\right)^{\lambda-1 / 2} d t\right]^{1 / p} .
\end{aligned}
$$

The $p$ th power of the first term is bounded by

$$
M \int_{-1+(1-r)^{2}}^{1-(1-r)^{2}}\left(1-t^{2}\right)^{(\alpha-2 \lambda-2) / 2}\left(1-t^{2}\right)^{\lambda-1 / 2} d t \leqq M^{\prime}(1-r)^{(\alpha-2 \lambda-2) p+2 \lambda+1} .
$$

Hence the first term is bounded by

$$
M^{\prime \prime}(1-r)^{(\alpha-2 \lambda-2)+(2 \lambda+1) p^{-1}}=M^{\prime \prime}(1-r)^{\alpha-\left(n / p^{\prime}\right)-1+\left(1 / p^{\prime}\right)}
$$

since $2 \lambda+1=(2 \lambda+1) / p+n / p^{\prime}-1 / p^{\prime}$ and thus $1 / p^{\prime}-n / p^{\prime}-1=$ $(2 \lambda+1) / p-2 \lambda-2$. For the last two terms we use the fact that $\left|Z_{e_{1}}^{(k)}(x)\right| \leqq B k^{2 \lambda}$. So the $p$ th power of the second or third terms is 
bounded by

$$
B^{\prime}\left(\sum_{k=1}^{\infty} k^{1-\alpha} k^{2 \lambda} r^{k}\right)^{p}(1-r)^{2 \lambda+1} \leqq B^{\prime \prime}(1-r)^{\langle\alpha-2 \lambda-2) p+2 \lambda+1}
$$

since $\sum_{k=1}^{\infty} k^{\beta} r^{k} \leqq A(1-r)^{-1-\beta}$ if $\beta>-1$. Hence, the second and third terms are bounded by

$$
B^{\prime \prime \prime}(1-r)^{\alpha-2 \lambda-2+(2 \lambda+1) p^{-1}}=B^{\prime \prime \prime}(1-r)^{\alpha-\left(n / p^{\prime}\right)-1+\left(1 / p^{\prime}\right)} .
$$

Thus $(1-r)^{1-\alpha+(n-1) / p^{\prime}}\left\|T G^{\alpha}(r x)\right\|_{p, d x} \leqq K$ if $p<\infty$. If $p=\infty$, as in the estimates for the second and third terms we have

$$
\left\|T G^{\alpha}(r x)\right\|_{\infty, d x} \leqq K(1-r)^{\alpha-2 \lambda-2}=K(1-r)^{\alpha-n} .
$$

Hence $(1-r)^{n-\alpha}\left\|T G^{\alpha}(r x)\right\|_{\infty, d x} \leqq K$. The result now follows from (5.2) and Proposition (5.1).

(b) If $\alpha \geqq 2 \lambda+2$, a similar argument applies.

$$
G^{\alpha}(x) \in \Lambda\left(\alpha-\frac{(n-1)}{p^{\prime}} ; p, \infty\right) \text { if } \alpha-\frac{n-1}{p^{\prime}}>0 \text {. }
$$

Proof. The proof is similar to the corresponding result by Taibleson [8; p. 428]. One can write $G^{\alpha}=G^{\alpha_{1} *} \ldots * G^{\alpha_{\bar{\alpha}}}$ where $\bar{\alpha}=\alpha_{1}+\cdots+\alpha_{\bar{\alpha}}$, $0<\alpha_{1}-(n-1) / p^{\prime}<1$, and $0<\alpha_{2}=\cdots=\alpha_{\bar{\alpha}}<1$. The result easily follows from (5.8).

Definition. For $\alpha>0$ and $f \in L_{p}\left(\Sigma_{n-1}\right), 1 \leqq p \leqq \infty$, define $J^{\alpha}$ by $J^{\alpha} f(x)=G^{\alpha *} f(x)$. Since $G^{\alpha} \in L_{1}\left(\Sigma_{n-1}\right)$, this convolution is well-defined.

Proposition 5.10. Let $\alpha, \beta>0,1 \leqq p, q \leqq \infty$. Then $J^{\beta}$ maps $\Lambda(\alpha ; p, q)$ isomorphically onto $\Lambda(\alpha+\beta ; p, q)$.

Proof. (a ) $J^{\beta}$ maps $\Lambda(\alpha ; p, q)$ continuously into $\Lambda(\alpha+\beta ; p, q)$. The proof is analogous to the proof of the corresponding part of Theorem 5 of Taibleson [19; p. 429]. The proof uses (5.9).

(b) $J^{\beta}$ maps $\Lambda(\alpha ; p, q)$ isomorphically onto $\Lambda(\alpha+\beta ; p, q)$. For $\beta>0$ and $f \in L_{p}\left(\Sigma_{n-1}\right)$ let

$$
J^{-\beta} f(r x)=G^{-\beta}(r, \cdot)^{*} f(x) .
$$

This is well-defined since for fixed $r<1, G^{-\beta}(r x)$ is a bounded function of $x$. It suffices to prove the proposition for $0<\beta<1 . \quad G^{-\beta}(r x)=$ $T G^{1-\beta}(r x)$ and $1>1-\beta>0$. By (5.9), $G^{1-\beta} \in \Lambda(1-\beta ; p, q)$. Again, proceeding as in the proof of Theorem 5 of Taibleson [19; p. 429], one can show that $J^{-\beta} f \in \Lambda(\alpha: p, q)$ for $f \in \Lambda(\alpha+\beta ; p, q)$. We need to show that $J^{-\beta}\left(J^{\beta} f\right)(x)=f(x)$ for almost every $x \in \Sigma_{n-1}$ if $f \in \Lambda(\alpha ; p, q)$. This 
follows from the fact that

$$
\left[G^{-\beta}\left(r_{1}, \cdot\right)^{*} G^{\beta}\left(r_{2}, \cdot\right)\right](x)=P\left(r_{1} r_{2} x \cdot e_{1}\right) .
$$

Similarly $J^{\beta}\left(J^{-\beta} f\right)(x)=f(x)$ for almost every $x \in \Sigma_{n-1}$ if $f \in \Lambda(\alpha+\beta$; $p, q)$. This finishes the proof of the proposition.

REMARK. In view of the above, it follows that $J^{\alpha}$ is an elliptic pseudodifferential operator of order $-\alpha$. See Seeley [14] for a more extensive discussion of these operators.

6. Further remarks. In this section we would like to discuss another way of defining Lipschitz spaces on $\Sigma_{n-1}$. It involves the use of local coordinates. For brevity we consider only the case $0<\alpha<1$ and $p=\infty=q$. The reader is referred to Ragozin [12].

Let $B=\bar{B}_{n-1}=\left\{z \in \boldsymbol{R}^{n}:|z| \leqq 1\right\}$. For $f \in L_{\infty}(B)$, we say $f \in \Lambda_{\alpha}(B)$ if the norm

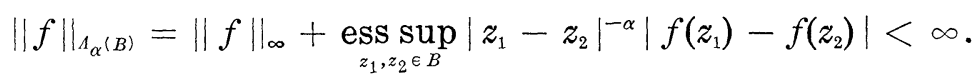

REMARK 6.1. The following are easily seen to be linear transforms of $\Lambda_{\alpha}(B)$ into itself:

(a) multiplication by $C^{\infty}$ functions and

(b) $f \mapsto f \circ \varphi$ where $\varphi: B \rightarrow B$ is any $C^{\infty}$ map.

We now define $\Lambda_{\alpha}\left(\Sigma_{n-1}\right)$ to be $\left\{f \in L_{\infty}\left(\Sigma_{n-1}\right)\right.$ : For each $x \in \Sigma_{n-1}$, there exists a $C^{\infty}$ chart $\varphi: B \rightarrow \Sigma_{n-1}$ such that $x \in \operatorname{int} \varphi(B)$ and $\left.f \circ \varphi \in \Lambda_{\alpha}(B)\right\}$.

REMARK 6.2. In view of Remark 6.1 , if $\varphi_{i}: B \rightarrow \Sigma_{n-1}, i=1, \cdots, n$, are a finite collection of $C^{\infty}$ charts with $\Sigma_{n-1}=\bigcup_{i=1}^{l}$ int $\varphi_{i}(B)$, then $f \in \Lambda_{\alpha}\left(\Sigma_{n-1}\right)$ if and only if $f \circ \varphi_{i} \in \Lambda_{\alpha}(B)$ for each $i$. Moreover, one can define a norm

$$
\|f\|_{\Lambda_{\alpha}\left(\Sigma_{n-1}\right)}=\sum_{i=1}^{l}\left\|f \circ \varphi_{i}\right\|_{\Lambda_{\alpha}(B)} .
$$

We would like to show that this definition is equivalent to $\Lambda(\alpha ; \infty$, $\left.\infty ; \Sigma_{n-1}\right)$. By virtue of Proposition 4.1, $\Lambda\left(\alpha ; \infty, \infty ; \Sigma_{n-1}\right)$ is the collection of functions for which the norm

$$
\|f\|_{\alpha: \infty, \infty}=\|f\|_{\infty}+\operatorname{ess}_{x, y \in \Sigma_{n-1}} \sup _{n-1} d(x, y)^{-\alpha}|f(x)-f(y)|<\infty .
$$

The following is well known.

REMARK 6.3. Let $\varphi$ be a $C^{\infty}$ chart: $B \rightarrow \Sigma_{n-1}$. Then (a) $d(x, y)$ is equivalent to $|x-y|$ for all $x, y \in \Sigma_{n-1}$, (b) $\left|\varphi\left(z_{1}\right)-\varphi\left(z_{2}\right)\right| \leqq M\left|z_{1}-z_{2}\right|$ for all $z_{1}, z_{2} \in B$, and (c) $\varphi^{-1}(x)-\varphi^{-1}(y)|\leqq M| x-y \mid$ for all $x, y \in \Sigma_{n-1}$. 
REMARK 6.4. In view of Remark 6.3, it easily follows that

$$
\underset{x, y \in \varphi(B)}{\operatorname{ess} \sup _{(B)}}\left(|f(x)|+d(x, y)^{-\alpha}|f(x)-f(y)|\right)<\infty
$$

if and only if $\|f \circ \varphi\|_{\Lambda_{\alpha}(B)}<\infty$. Thus, the two definitions agree locally. One can then use the Lebesgue number for the covering $\operatorname{int} \varphi_{1}(B), \cdots$, int $\left.\varphi_{l}(B)\right\}$ to show that the definitions agree globally. Let $\varepsilon>0$ be such that if $x, y \in \Sigma_{n-1}$ and $d(x, y)<\varepsilon$, then there exists $i$ such that $x \in \operatorname{int} \varphi_{i}(B)$. Then if $x, y \in \Sigma_{n-1}$ and $d(x, y) \geqq \varepsilon,|f(x)-f(y)| \leqq 2\|f\|_{\infty}=$ $2\left(\|f\|_{\infty} / \varepsilon^{\alpha}\right) \varepsilon^{\alpha} \leqq M d(x, y)^{\alpha}$.

It is easy enough to see that the norms corresponding to the two definitions are equivalent also.

\section{REFERENCES}

1. R. Askey and S. Wainger, On the behavior of special classes of ultraspherical expansions-I, J. D'Analyse Mathématique, 15 (1965), 193-220.

2. H. Bateman, Higher Transcendental Functions, Vol. 2, McGraw-Hill, New York, Toronto, London, 1953.

3. A. P. Calderón, Intermediate spaces and interpolation, the complex method, Studia Math., 24 (1964), 113-190.

4. A. P. Calderón and A. Zygmund, On a problem of Mihlin, Trans. Amer. Math. Soc., 78 (1955), 209-224.

5. R. R. Coifman and G. L. Weiss, Representations of compact groups and spherical harmonics, L'Enseignment Mathématique, Tome XIV, fasc. 2 (1968), 121-173.

6. G. H. Hardy, J. E. Littlewood, and G. Pólya, Inequalities, Cambridge, 1959.

7. N. J. H. Heideman, Duality and fractional integration in the spaces $A(B, X)$ of

A. P. Calderón, Ph. D. dissertation, Washington University, (1970).

8. I. I. Hirschman, Jr., Fractional Integration, Amer. J. Math., 75 (1953), 531-546.

9. S. M. Nikolśkiî, On imbedding, continuation and approximation theories for differentiable functions of several variables, Russian Math. Surveys, London Math. Soc., 16, No. 5 (1961), 55-104.

10. J. Peetre, Espaces d'Interpolation et Théoreme de Soboleff, Ann. Inst. Fourier, Grenoble 16, 1 (1966), 279-317.

11. D. L. Ragozin, Polynomial Approximation and Harmonic Analysis on Spheres, Seminar Report, Mass. Inst. of Tech., 1968.

12. - Polynomial approximation on compact manifolds and homogeneous spaces, Trans. Amer. Math. Soc., 150 (1970), 41-53.

13. R. T. Seeley, Spherical Harmonics, No. 11 of the H. Ellsworth Slaught Memorial Papers, Amer. Math. Monthly, 73, No. 4 (1966), 115-121.

14. Complex powers of an elliptic operator, Proc. Symp. Pure Math., 10 (1967), 288-307.

15. E. M. Stein, On the functions of Littlewood-Paley, Lusin and Marcinkiewicz, Trans. Amer. Math. Soc., 88 (1958), 430-466.

16. - Topics in Harmonic Analysis Related to the Littlewood-Paley Theory, Annals of Mathematics Studies, No. 63, Princeton University Press, 1970.

17. E. M. Stein and G. L. Weiss, Introduction to Fourier Analysis on Euclidean Spaces, Princeton University Press, 1971.

18. M. H. Taibleson, On the theory of Lipschitz spaces of distributions on Euclidean n-space, I, Principal properties, J. Math. and Mech., 13, No. 13 (1964), 407-480.

19. - II, Translation invariant operators, duality, and interpolation, J. Math. and Mech., 14, No. No. 5, (1965), 821-840. 
20. M. H. Taibleson, III, Smoothness and integrability of Fourier transforms, smoothness of convolution kernels, J. Math. and Mech., 15, No. 6 (1966), 973-982.

21. A. Zygmund, Trigonometric Series, Sec. Ed., Cambridge, 1968.

Received June 28, 1971 and in revised form June 5, 1973.

UNIVERSITY OF CALIFORNIA, IRVINE 


\section{PACIFIC JOURNAL OF MATHEMATICS}

\section{EDITORS}

RICHARD ARENS (Managing Editor) University of California

Los Angeles, California 90024

R. A. BeaUmont

University of Washington Seattle, Washington 98105
J. DUGUNDJI*

Department of Mathematics University of Southern California Los Angeles, California 90007

D. Gilbarg and J. Milgram Stanford University

Stanford, California 94305

\section{ASSOCIATE EDITORS}

E. F. BECKENBACH

B. H. NeUmanN

F. WOLF

K. YoSHIDA

\section{SUPPORTING INSTITUTIONS}

\section{UNIVERSITY OF BRITISH COLUMBIA CALIFORNIA INSTITUTE OF TECHNOLOGY UNIVERSITY OF CA.LIFORNIA MONTANA STATE UNIVERSITY UNIVERSITY OF NEVADA NEW MEXICO STATE UNIVERSITY OREGON STATE UNIVERSITY UNIVERSITY OF OREGON OSAKA UNIVERSITY}

UNIVERSITY OF SOUTHERN CALIFORNIA STANFORD UNIVERSITY UNIVERSITY OF TOKYO UNIVERSITY OF UTAH WASHINGTON STATE UNIVERSITY UNIVERSITY OF WASHINGTON AMERICAN MATHEMATICAL SOCIETY NAVAL WEAPONS CENTER

* C. R. DePrima California Institute of Technology, Pasadena, CA 91109, will replace J. Dugundji until August 1974. 


\section{Pacific Journal of Mathematics}

\section{Vol. 50, No. $1 \quad$ September, 1974}

Gail Atneosen, Sierpinski curves in finite 2-complexes.............. 1

Bruce Alan Barnes, Representations of $B^{*}$-algebras on Banach spaces .... 7

George Benke, On the hypergroup structure of central $\Lambda(p)$ sets ....... 19

Carlos R. Borges, Absolute extensor spaces: a correction and an

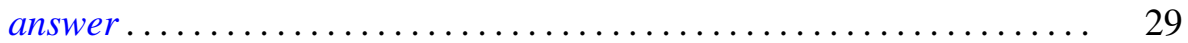

Tim G. Brook, Local limits and tripleability .................. 31

Philip Throop Church and James Timourian, Real analytic open maps .... 37

Timothy V. Fossum, The center of a simple algebra ............... 43

Richard Freiman, Homeomorphisms of long circles without periodic

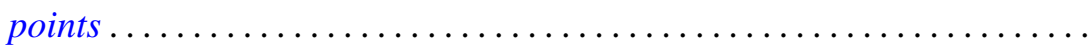

B. E. Fullbright, Intersectional properties of certain families of compact

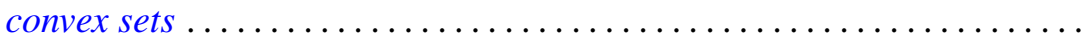

Harvey Charles Greenwald, Lipschitz spaces on the surface of the unit

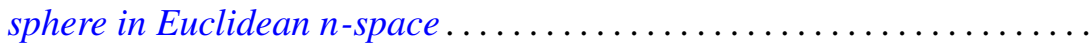

Herbert Paul Halpern, Open projections and Borel structures for

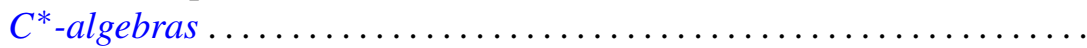

Frederic Timothy Howard, The numer of multinomial coefficients divisible

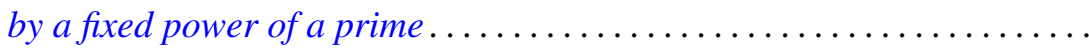

Lawrence Stanislaus Husch, Jr. and Ping-Fun Lam, Homeomorphisms of manifolds with zero-dimensional sets of nonwandering points........ 109

Joseph Edmund Kist, Two characterizations of commutative Baer rings ...

Lynn McLinden, An extension of Fenchel's duality theorem to saddle functions and dual minimax problems ................

Leo Sario and Cecilia Wang, Counterexamples in the biharmonic classification of Riemannian 2-manifolds...

Saharon Shelah, The Hanf number of omitting complete types ...

Richard Staum, The algebra of bounded continuous functions into a

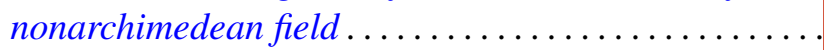

James DeWitt Stein, Some aspects of automatic continuity ..

Tommy Kay Teague, On the Engel margin

John Griggs Thompson, Nonsolvable finite groups all of whose local subgroups are solvable, $V \ldots \ldots \ldots \ldots \ldots \ldots \ldots \ldots$

Kung-Wei Yang, Isomorphisms of group extensions 